\title{
Tendências das Publicações nas Fases Trimestral, Bimestral e Mensal das Cinco Décadas dos Arquivos Brasileiros de Cardiologia
}

\author{
Alfredo José Mansur, Adriana Salatini Abud, Cícero Piva de Albuquerque
}

São Paulo, SP

\begin{abstract}
Objetivo - Estudar tendências das publicações nos Arquivos Brasileiros de Cardiologia de março de 1948 a fevereiro de 1998, nas fases trimestral, bimestral e mensal de publicação.
\end{abstract}

Métodos - Foram sorteados aleatoriamente $25 \%$ dos fascículos de cada fase e constituída amostra de 98 fascículos: $13(11,5 \%)$ da fase trimestral, 27 (23,5\%) da fase bimestral e 58(65\%) da fase mensal. Foram estudados o tipo de texto, o número de autores, a procedência, o idioma, $e$ as referências bibliográficas.

Resultados - Foram estudados 1.204 textos, 90 (7,5\%) da fase trimestral, $238(19,8 \%)$ da fase bimestral e 876 $(72,8 \%)$ dafase mensal. Os textos mais freqüentes foram artigos originais (353), revisões de tema (350) e relatos de caso (205). Nas três fases de periodicidade: não houve diferença significativa das proporções de artigos originais, revisões $e$ relatos de caso; houve maior número de autores na fase mensal; verificou-se concentração geográfica das contribuições [864 de 1204 (72\%) de três Estados], diminuíram as contribuições internacionais (20,8\%, 4,4\% e 4,9\%), houve redução de publicações em outras línguas; a média de referências internacionais superou 16,7 e de nacionais foi inferior a 4,7.

Conclusão - A análise da tendência evolutiva em cinco décadas de publicação revelou hiatos que podem ser preenchidos pelos Arquivos Brasileiros de Cardiologia, para melhor acompanhar as tendências internacionais de editoração de periódicos médicos, das expectativas dos autores e dos leitores.

Palavras-chave: Periódicos/Padrões; Publicação.
Publication trends in the quartely, bimonthly and monthly cycles of publication in the Brazilian Archives of Cardiology over five decades

Objective - To study trends in selected manuscript characteristics of articles published in the Brazilian Archives of Cardiology from March 1948, to February 1998, in the quarterly, bimonthly and monthly cycles of publication.

Methods - A random sample of $25 \%$ of all issues of the journal comprised the study sample: 13 issues (11.5\%) from the quarterly, 27 (23,5\%) from the bimonthly, and 58 (65\%) from the monthly publication cycle. We studied the type of manuscript, number of authors, geographical distribution, language of publication and references.

Results - A total of 1204 articles were studied, 90 (7.5\%) from the quarterly, 238 (19,8\%) from the bimonthly, and $876(72.8 \%)$ from the monthly publication cycle. The most frequent published articles were original contributions (353), reviews (350) and case reports (205). No significant difference occurred in the proportion of original articles, reviews and case reports; the number of authors was higher in the monthly period; a geographical concentration of the contributions occurred (72\% from three Brazilian States); manuscripts in languages other than Portuguese decreased. The mean number of Brazilian references cited was less than 4.7 and the mean number of international references cited was greater than 16.7.

Conclusion - The analysis of the trends over five decades of publication revealed the need for further steps to be taken by the Brazilian Archives of Cardiology, to meet international publication standards for biomedical journals as well as authors' and readers' demands.

Key words: Periodicals/Standards; Publishing.

Arq Bras Cardiol, volume 75 (n 1), 1-7, 2000

Instituto do Coração do Hospital das Clínicas - FMUSP

Correspondência: Alfredo José Mansur - InCor - Unidade Clínica de Ambulatório Geral - Av. Dr. Enéas C. Aguiar, 44 - 05403-000 - São Paulo, SP e-mail: ajmansur@ incor.usp.br Recebido para publicação em 24/5/00

Aceito em 28/6/00
Arquivos Brasileiros de Cardiologia foram publicados com periodicidade trimestral a partir de 1948, passaram à periodicidade bimestral em 1961, e são publicados mensalmente desde 1979. Parte da fase bimestral (1968-1977) foi examinada há 20 anos, tanto no que diz respeito à sua inser- 
ção cultural, quanto nos aspectos bibliométricos; reiterouse na ocasião a existência de literatura cardiológica nacional consistente e foram sugeridos pontos para reflexão ${ }^{1}$. Recentemente, Arquivos foram considerados fonte relevante de informação por $74 \%$ de 1750 cardiologistas brasileiros entrevistados em congresso de cardiologia ${ }^{2}$.

Reflexões fazem parte do processo editorial ${ }^{1,3-5}$, que pode ser entendido como um continuum da atividade científica ${ }^{6}$ com reuniões, congressos, publicações, revisão pelos pares ${ }^{7} \mathrm{e} \operatorname{cartas}^{8}$. Atualmente, há pletora de revistas ${ }^{5} \mathrm{e}$ de informação médica ${ }^{6}$. Há também correlação entre o número de revistas de uma área e os valores máximo, médio e mínimo do fator de impacto das revistas deste grupo ${ }^{9}$. Portanto, no âmbito cardiológico, as exigências para uma revista desfrutar de elevada reputação são rigorosas.

Os objetivos dos Arquivos foram expressos no editorial de inauguração em março de 1948: sob os auspícios da Sociedade Brasileira de Cardiologia, incentivar estudos e pesquisas cardiológicas no Brasil, em alto nível científico, e ter suas páginas abertas a todos que contribuem para o progresso da Cardiologia ${ }^{10}$. Os objetivos de revistas médicas são continuamente avaliados na literatura, e incluem: ser fonte independente de idéias e reflexões ${ }^{11}$, ser foro de discussão da prática médica ${ }^{6}$; ter o clínico atuante como o público alvo ${ }^{5}$, levar aos leitores a fascinação de descobrirem novos temas e informações que não eram esperadas ${ }^{5}$,e estimular leitores a refletirem ${ }^{12}$, ser um processo educativo tanto para os autores como para os revisores ${ }^{13}$, traduzir conhecimento básico em clínico e compartilhar experiência clínica ${ }^{14}$, ser fonte de referência para médicos, pacientes e familiares ${ }^{15}$, e contribuir para a educação de pacientes ${ }^{16}$. Há revistas que optaram por uma filosofia editorial, como por exemplo, luta contra o tabagismo, contra o porte de armas, ou a favor da medicina baseada em evidências ${ }^{12}$. Portanto, os objetivos de revistas médicas são bastante abrangentes.

A revista deve atender tanto os pesquisadores que encaminham artigos para publicação, quanto os seus leitores. Os objetivos principais dos pesquisadores na publicação são: a) validar resultados (validação essa que depende da reputação da revista); b) chegar ao público alvo (em geral tão internacional quanto possível), e c) alcançar reconhecimento pela excelência do trabalho ${ }^{17}$. Cardiologistas brasileiros que publicam estão mais freqüentemente ligados a programas de pós-graduação ${ }^{18}$, os quais pressionam por melhores perfis de publicações, com visibilidade internacional ${ }^{19}$. Nesse contexto, o fator de impacto (número de citações em relação ao número de publicações na revista nos dois anos anteriores ${ }^{20}$ é relevante para a reputação do periódico e pode ser entendido como indicador de qualidade, embora não reflita a qualidade de um trabalho ou de um pesquisador em particular ${ }^{21}$. As revistas com baixo índice de impacto sofrem evasão de pesquisadores ${ }^{9,22}$, inclusive os de maior reputação ${ }^{9}$; desse modo, revistas com baixo fator de impacto tendem à inércia, sem condições de se fortalecerem ${ }^{9}$. O caráter heterogêneo da qualidade das comunicações foi apontado como um dos pesos da balança de competitividade dos Arquivos para se habilitarem a receber contribuições ${ }^{1}$. Em questionário aplicado a 479 pesquisadores da Faculdade de Medicina de Stanford, as duas características mais importantes que influenciaram na escolha de periódico para submeter um trabalho com vistas à publicação foram o prestígio científico (do qual o fator de impacto é uma indicação) e o número de leitores, e as duas menos importantes o editor ser conhecido e a atenção da imprensa leiga ${ }^{23}$.

Igualmente relevante é a opinião dos leitores. Por exemplo, de dez temas hierarquizados entre os mais relevantes pelos membros de conselho editorial, apenas três foram assim hierarquizados pelos leitores ${ }^{24}$, e segundo pesquisa espanhola, os leitores preferem artigos de revisão a outros tipos de artigo ${ }^{22}$. Portanto, a opinião do conselho editorial e a dos leitores não são sempre superponíveis. É possível que a tensão desse hiato seja saudável e até mesmo necessária do contexto educativo, pré-requisito para a troca de idéias do processo editorial. Este deve também contribuir para prevenir a banalização da prática médica, para menor imediatismo no processo de educação contínua, em tempos de hiperpragmatismo operacional acrítico, e para ampliar horizontes culturais. Os leitores não devem ser subestimados com pressuposições de indiferença frente a temas relevantes ${ }^{25}$.

Tendo em vista as transformações científicas e da prática médica ${ }^{1,4}$, estudamos aspectos selecionados das cinco décadas de publicação dos Arquivos Brasileiros de Cardiologia, com base nas suas diferentes periodicidades. Pretendemos desse modo contribuir para reflexões adicionais sobre a melhor orientação editorial, no contexto das normas internacionais de edição, da expectativa dos autores e dos leitores.

\section{Métodos}

Critério amostral - Trezentos e noventa fascículos publicados de março de 1948 a fevereiro de 1998 foram categorizados de acordo com a periodicidade trimestral, bimestral ou mensal da publicação. Em seguida, foram sorteados aleatoriamente $25 \%$ dos 52 da fase trimestral, $25 \%$ dos 108 da fase bimestral e $25 \%$ dos 230 da fase mensal. Assim foi constituída a amostra de estudo de 98 fascículos: $13(11,5 \%)$ da fase trimestral, $27(23,5 \%)$ da fase bimestral e $58(65 \%)$ dafase mensal.

Variáveis estudadas - a) tipos de texto: foi adotada a categorização da Revista e os textos foram agrupados em: a) artigo original, b) revisão de tema: editorial, conferência, tese, discurso, comentário editorial, ponto de vista, nota prévia, atualização, simpósio, caminhos e história da cardiologia, c) relato de caso: até cinco casos descritos, correlação anatomoclínica, descrição patológica, discussão de caso; d) estudo experimental; e) ensaio terapêutico, ação medicamentosa, f) estudo estatístico, estudo epidemiológico; g) revisão de livro ou revista, h) carta ao editor; i) in memoriam, homenagem, oração; j) noticiário, noticiário Funcor; k) outros: testes, estatutos da Sociedade Brasileira de Cardiologia, reformas de estatuto. As informações publicitárias não foram avaliadas; b) número de autores por arti- 
go; c) procedência das publicações: foram caracterizados em: a) estado nacional brasileiro, b) internacional, c) multicêntrica nacional, e d) multicêntrica internacional; d) idioma da publicação; e) referências bibliográficas: categorizadas em: a) total, b) nacional e c) internacional.

Análise estatística -Foi utilizado o software EPIinfo, para constituir a base de dados com as amostras coletadas em formato de questionário ${ }^{26}$. Os resultados foram obtidos com o programa Analysis, comandos freq e means. A comparação de proporções foi feita com o emprego do teste de $\chi^{2}$. Valores de $\mathrm{p}<0,05$ foram adotados como estatisticamente significantes.

\section{Resultados}

Foram estudados 1.204 textos, $90(7,5 \%)$ da fase trimestral, $238(19,8 \%)$ da fase bimestral e $876(72,8 \%)$ da fase mensal.

a) Tipos de textos - Os textos mais freqüentes foram os artigos originais, seguidos de revisões de tema e de relatos de caso (tab. I). A comparação de proporções desses três tipos de texto nas fases trimestral, bimestral e mensal não revelou diferença significativa $\left(\chi^{2}=8,765, \mathrm{p}=0,067\right)$. Onúmero de cartas ao editor foi pequeno; b) número de autores - a comparação das proporções de 1 a 2 autores, 3 a 4, 5 a 6 e mais de 6 autores nas três fases de periodicidade revelou diferença estatísticamente significante $\left(\chi^{2}=46,5, \mathrm{p}=0,001\right)$. Artigos com um ou dois autores diminuíram no suceder das três fases de publicação, e aumentaram os artigos com cinco ou seis autores (tab. II). Artigos com mais de seis autores apareceram e foram mais freqüentes na fase bimestral de publicação; c) procedência das publicações - houve maior contribuição de diferentes regiões geográficas do país no suceder das três fases de publicação. O exame do total revelou concentração regional de publicações, principalmente nos Estados de São Paulo, Rio de Janeiro e Rio Grande do Sul. A comparação das proporções das contribuições de São Paulo, do Rio de Janeiro, de outros estados, e internacionais nas três fases de periodicidade revelou diferença significativa $\left(\chi^{2}=40,348 ; p=0,001\right)$, que pode ser atribuída à maior proporção de publicações internacionais na fase trimestral $\left(\chi^{2}=24,73 ; p=0,001\right)$, com diminuição percentual

\begin{tabular}{|c|c|c|c|c|c|c|c|c|}
\hline \multirow[t]{3}{*}{ Tipo de texto } & \multicolumn{8}{|c|}{ Fases de periodicidade } \\
\hline & \multicolumn{2}{|c|}{ Trimestral } & \multicolumn{2}{|c|}{ Bimestral } & \multicolumn{2}{|c|}{ Mensal } & \multicolumn{2}{|c|}{ Total } \\
\hline & $\mathrm{n}$ & $(\%)$ & $\mathrm{n}$ & $(\%)$ & $\mathrm{n}$ & $(\%)$ & $\mathrm{n}$ & $(\%)$ \\
\hline Artigo original & 15 & 16,7 & 65 & 27,3 & 273 & 31,2 & 353 & 29,3 \\
\hline Carta & & & 2 & 0,8 & 35 & 4 & 37 & 3,1 \\
\hline Estudo estatístico & 8 & 8,9 & 3 & 1,3 & 23 & 2,6 & 34 & 2,8 \\
\hline Ensaio terapêutico & 4 & 4,4 & 22 & 9,2 & 58 & 6,5 & 84 & 7 \\
\hline Estudo experimental & 6 & 6,7 & 9 & 3,8 & 27 & 3,1 & 42 & 3,5 \\
\hline In memoriam & 2 & 2,2 & 3 & 1,3 & 4 & 0,5 & 9 & 0,7 \\
\hline Noticiário & 11 & 12,2 & 20 & 8,4 & 35 & 4 & 66 & 5,5 \\
\hline Outros & & & 3 & 1,3 & 11 & 1,3 & 14 & 1,2 \\
\hline Relato de caso & 12 & 13,3 & 30 & 12,6 & 163 & 18,6 & 205 & 17 \\
\hline Revisão de livro & 5 & 5,6 & 5 & 2,1 & & & 10 & 0,8 \\
\hline Revisão de tema & 27 & 30 & 76 & 31,9 & 247 & 28,2 & 350 & 29,1 \\
\hline Total & 90 & 100 & 238 & 100 & 876 & 100 & 1204 & 100 \\
\hline
\end{tabular}

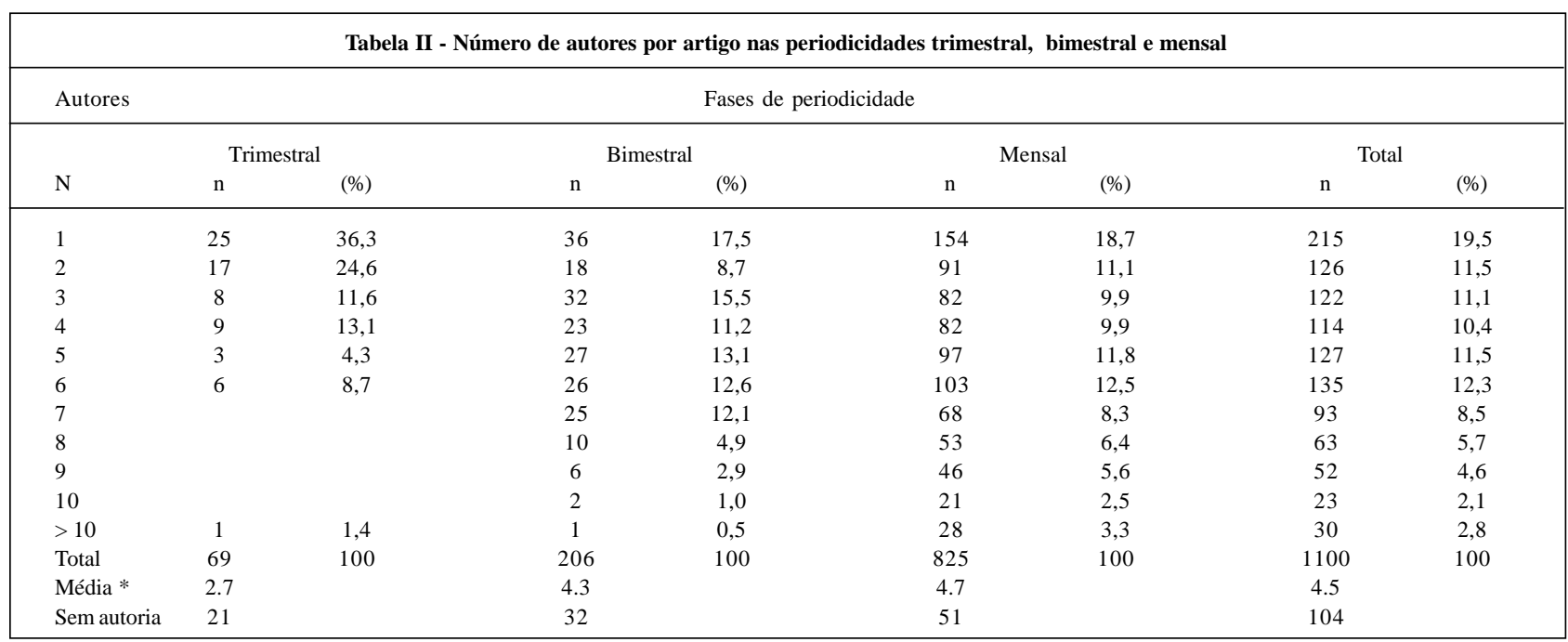


com o passar do tempo (tab. III); d) idioma - $10 \%$ das publicações do período trimestral foram em língua estrangeira (inglês, espanhol e francês), 6,8\% na fase bimestral e 3,0\% na fase mensal (tab. IV). A comparação de proporções das publicações em português e em outras línguas nas três fases de periodicidade revelou diferença significativa $\left(\chi^{2}=13,599\right.$; $\mathrm{p}=0,001)$; e) número de referências bibliográficas - as referências internacionais excedem as nacionais (tab. V).

\section{Discussão}

Os artigos originais são o foco de maior interesse dos Arquivos Brasileiros de Cardiologia, assim como de outras revistas ${ }^{14,15}$, pois são lídimas expressões da geração de conhecimento, contribuem para melhorar os Serviços nos quais são realizados e transformam os processos de sofrimento e doença dos pacientes, em contribuição para o conhecimento médico, e por meio da atuação médica, em contribuição para a sociedade, tanto mais ampla quanto mais amplo for o alcance da revista. Nos Arquivos, os artigos originais e revisões foram eqüifreqüentes. Seria desejável atender as exigências atuais de qualificação das revistas médicas, tanto por parte de fontes indexadoras ${ }^{20}$, quanto por parte dos núcleos de pós-graduação e dos próprios autores ${ }^{19}$, priorizando as contribuições originais. A comparação das fases trimestral, bimestral e mensal não revelou modificação significativa das proporções de artigos originais, revisões de tema e relatos de caso, tendência que sugere pouco dinamismo. Cabe a indagação se essa tendência exprime a dinâmica editorial da revista, ou da cultura que lhe dá origem. O número de artigos originais brasileiros não aumentou de 1981 a 1993 nas revistas nacionais indexadas no Institute of Scientific Information ${ }^{19}$, o qual ainda não inclui os Arquivos Brasileiros de Cardiologia.

O estímulo mais ativo para que maior contingente das 400 apresentações de congressos anuais da Sociedade Brasileira de Cardiologia se transforme em artigos originais é método factível de trabalho. Essa iniciativa foi reconhecida como do escopo de sociedades médicas ${ }^{27}$. $\mathrm{O}$ aumento do número de contribuições é importante, pois a partir de um certo número mínimo de artigos, deixa de haver grandes oscilações de fator de impacto ${ }^{21}$. A taxa de rejeição de artigos

\begin{tabular}{|c|c|c|c|c|c|c|c|c|}
\hline \multirow[t]{2}{*}{ Procedência } & \multicolumn{8}{|c|}{ Fases de Periodicidade } \\
\hline & $\begin{array}{c}\text { Trimestral } \\
\mathrm{n}\end{array}$ & $\begin{array}{c}\text { Trimestral } \\
(\%)\end{array}$ & $\begin{array}{c}\text { Bimestral } \\
\mathrm{n}\end{array}$ & $\begin{array}{c}\text { Bimestral } \\
(\%)\end{array}$ & $\begin{array}{c}\text { Mensal } \\
\mathrm{n}\end{array}$ & $\begin{array}{c}\text { Mensal } \\
(\%)\end{array}$ & $\begin{array}{c}\text { Total } \\
\mathrm{n}\end{array}$ & $\begin{array}{c}\text { Total } \\
(\%)\end{array}$ \\
\hline \multicolumn{9}{|l|}{ Nacional } \\
\hline Alagoas & & & & & 1 & 0,1 & 1 & 0,1 \\
\hline Bahia & 5 & 6,9 & 2 & 1,0 & 11 & 1,3 & 18 & 1,6 \\
\hline Ceará & 1 & 1,4 & 1 & 0,5 & 7 & 0,9 & 9 & 0,8 \\
\hline Distrito Federal & & & 1 & 0,5 & 5 & 0,6 & 6 & 0,5 \\
\hline Espírito Santo & & & 2 & 1,0 & 8 & 1,0 & 10 & 0,9 \\
\hline Goiás & & & & & 12 & 1,5 & 12 & 1,1 \\
\hline Minas Gerais & & & 3 & 1,5 & 27 & 3,3 & 30 & 2,7 \\
\hline Mato Grosso do Sul & & & & & 1 & 0,1 & 1 & 0,1 \\
\hline Pará & & & & & 1 & 0,1 & 1 & 0,1 \\
\hline Pernambuco & 3 & 4,2 & 2 & 1,0 & 25 & 3,0 & 30 & 2,7 \\
\hline Paraná & 1 & 1,4 & 4 & 2,0 & 17 & 2,1 & 22 & 2,0 \\
\hline Rio de Janeiro & 8 & 11,1 & 27 & 13,2 & 106 & 12,9 & 141 & 12,9 \\
\hline Rio Grande do Sul & 2 & 2,8 & 11 & 5,4 & 67 & 8,2 & 80 & 7,3 \\
\hline Santa Catarina & 1 & 1,4 & & & 1 & 0,1 & 2 & 0,2 \\
\hline Sergipe & & & & & 4 & 0,5 & 4 & 0,4 \\
\hline São Paulo & 35 & 48,6 & 141 & 69,1 & 467 & 57,0 & 643 & 58,7 \\
\hline $\begin{array}{l}\text { Multicêntrico } \\
\text { Internacional }\end{array}$ & & & & & 15 & 1,8 & 15 & 1,4 \\
\hline Multicêntrico & 1 & 1,4 & 1 & 0,5 & 5 & 0,6 & 7 & 0,6 \\
\hline Outros artigos & 15 & 20,8 & 9 & 4,4 & 40 & 4,9 & 64 & 5,8 \\
\hline
\end{tabular}

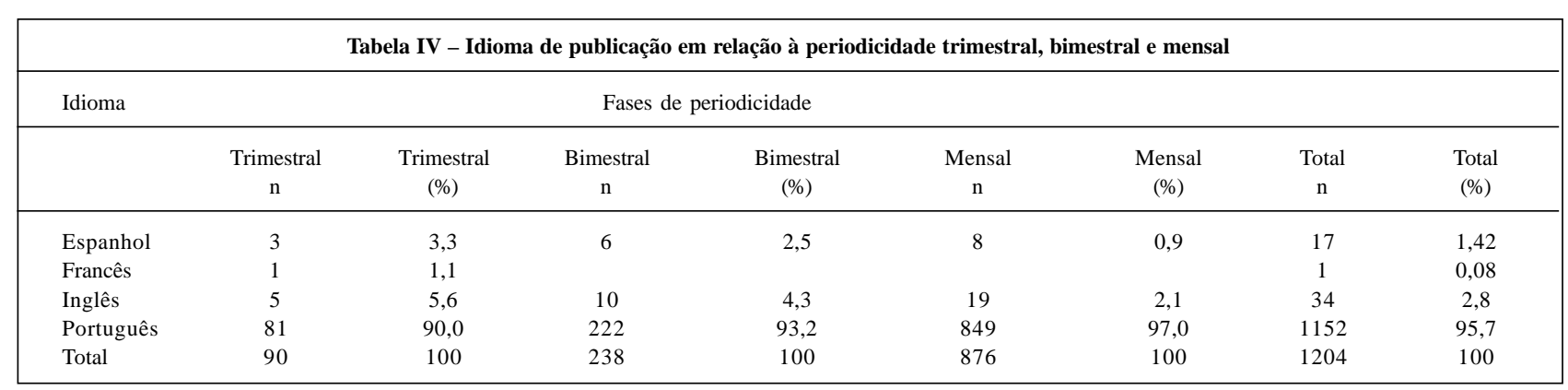




\begin{tabular}{|c|c|c|c|c|}
\hline \multicolumn{5}{|c|}{$\begin{array}{l}\text { Tabela } \mathrm{V} \text { - Médias das referências por artigo em relação à } \\
\text { periodicidade trimestral, bimestral e mensal }\end{array}$} \\
\hline \multicolumn{2}{|c|}{ Média das referências } & \multicolumn{3}{|c|}{ Fases de periodicidade } \\
\hline & Trimestral & Bimestral & Mensal & Total \\
\hline Nacional & 4,7 & 3,0 & 4,0 & 3,8 \\
\hline Internacional & 18,0 & 16,7 & 21,5 & 20,4 \\
\hline Total & 20,8 & 18,7 & 24,2 & 22,9 \\
\hline
\end{tabular}

nos Arquivos é consonante com a observação sobre o fator de impacto. Em 1995 as diferentes regiões geográficas do país tiveram taxas de recusa entre $20 \%$ a 50\%. A região com menor taxa de rejeição submeteu 59 trabalhos, e a região com maior taxa de rejeição submeteu dois artigos [Cardoso $\mathrm{G}$, comunicação pessoal].

O fato de apresentações de congressos freqüentemente não resultarem em publicação foi apontado no editorial dos Arquivos em março de $1956^{28}$. Uma das etapas do retardo é o pesquisador não submeter o $\operatorname{artigo~}^{29}$, mesmo no contexto de pressão por publicação ${ }^{30}$. Há a recomendação de não citar resumos de congresso como referência bibliográfica $^{31}$. Dos trabalhos apresentados em congresso da Sociedade Americana para Medicina de Urgência, 55\% não foram publicados em cinco anos e $20 \%$ deles não haviam sido submetidos ${ }^{27}$. Dos trabalhos apresentados em congressos cardiológicos $49,6 \%$ resultaram em publicações depois de 14 meses em média ${ }^{31}$. Entre as razões para a não publicação foram apontadas: falta de tempo (42\%), pressuposição de recusa (20\%), resultados considerados sem importância (12\%), problemas com co-autores (9\%), o trabalho "não valia a pena" (7\%), resultados semelhantes em outras publicações (6\%), ausência de resultados positivos (4\%), e outras $(22 \%)^{27}$. Curiosamente, o pessimismo (suposição de recusa pela revista) foi maior nas instituições de maior destaque ${ }^{27}$.

Discute-se a propriedade científica da publicação de relatos de caso. Em 1995, o Lancet reiniciou a publicação de relatos de caso, convidando especialmente os mais jovens para contribuírem ${ }^{32}$, mas há rejeição a esse tipo de publicação ${ }^{22,33}$. Considerando os Arquivos, nossa opinião é que deve haver espaço para essas publicações. Entretanto, o espaço editorial destinado a este tipo de publicação talvez tenha sido excessivo nos Arquivos (na fase mensal representou $18,6 \%$ dos artigos).

A publicação de revisões e atualizações -Arquivos recebem mais revisões do que podem absorver - parece ser do gosto de um certo público leitor, e o mesmo se verificou entre os leitores da Revista Espanhola de Cardiologia ${ }^{22}$. Se por um lado podem agradar a leitores, elas não contribuem para a qualificação da Revista e não estimulam os Serviços a gerarem dados próprios edificadores da cultura médica. Há no país muitas revistas dedicadas a revisões, que não deixam os autores sem veículo de expressão. Embora Arquivos devam acolher revisões, há consenso que deveriam ser revisões mediante convite, de autores que tem dados, reconhecida experiência ou notório saber crítico sobre o tema que abordam.
Também foi pequeno o número de cartas, consideradas revisão pelos pares a posteriori ${ }^{8}$, e expressão de leitura ativa pelos leitores ${ }^{15}$. Verifica-se que esse número pode e deve se ampliar.

O aumento do número de autores por artigo no decorrer do tempo, tendência da literatura internacional ${ }^{3,20}$, ocorreu mais em algumas revistas do que em outras ${ }^{34}$, tem causas múltiplas, entre elas a complexidade e a transdisciplinaridade da prática médica atual ${ }^{35,36}$, e também critérios mais elásticos de autoria ${ }^{35,37}$. A decisão do Comitê Internacional de Editores de Revistas Médicas de nomear o máximo de seis autores nas referências reduziu o número de artigos com sete autores ${ }^{38}$. Critérios de autoria foram sugeridos ${ }^{39}$, mas o assunto é mais do âmbito institucional do que da revista ${ }^{34}$, e objeto de contínua discussão ${ }^{34,35,40-42}$. Há revistas que limitam a sete autores de uma mesma instituição ${ }^{43}$, ou a três (Heart, International Journal of Cardiology) ou quatro autores nos relatos de caso ${ }^{32}$. Portanto, tanto revisores dos Arquivos, como a literatura internacional delineiam diretrizes que podem ser úteis.

O exame das procedências dos artigos publicados nos Arquivos revelou disparidade regional. No período de 1968 a 1977 a cidade de São Paulo foi responsável por cerca de $60 \%$ dos 659 artigos ${ }^{1}$. Na fase bimestral completa, do total de 238 textos publicados, 69,1\% eram do Estado de São Paulo, e na fase mensal mais recente, 57\% (tab. IV). Apesar disso, há a tendência de mais regiões geográficas serem representadas na fase mensal mais recente. Em países menores a concentração regional é mais compreensível ${ }^{44,45}$ do que no Brasil. Os Arquivos devem ser ambiente de ampliação da geografia das publicações médicas no país.

O número de publicações internacionais permaneceu no mesmo patamar em relação à fase bimestral ${ }^{1}$. Essa é uma tendência relevante, pois a internacionalidade, além de exigência editorial ${ }^{20}$ é o sentido para o qual se inclinam várias revistas ${ }^{25,46}$. Richard Smith, editor do British Medical Journal, comentou o paradoxo - ser ao mesmo tempo britânico e internacional ${ }^{5}$.É possível que seja uma demanda cultural de nossa época, ser simultaneamente uma publicação brasileira e internacional.

A pluralidade lingüística (ou internacionalidade) dos Arquivos foi marcante na fase inicial, cultivada como um progresso ${ }^{47}$ mas não se preservou. $\mathrm{O}$ alcance restrito dos Arquivos em razão da publicação em português já foi apontado há 20 anos ${ }^{1}$. O número atual de citações de artigos publicados nos Arquivos por autores que publicaram em outras revistas indexadas, mesmo sobre doenças por muito tempo endêmicas em nosso país, é pequeno. A publicação em língua portuguesa foi indicada como o principal fator de discriminação ${ }^{48}$, entre as tendenciosidades possíveis na revisão pelos pares, relacionadas à nacionalidade e língua, entre outras ${ }^{13}$. Em alguns países, a publicação na língua local foi considerada folclore ${ }^{49}$, e se no Brasil os críticos apontamos o "portinglês", na Holanda o mesmo fenômeno recebeu o nome de "dunglish" ("dutch" + "english") ${ }^{49}$. Por outro lado, na revista de instituição que já originara oito prêmios Nobel, a mudança do francês para o inglês não elevou 
o fator de impacto, mas o periódico previamente publicado em francês, atualmente o é em inglês ${ }^{50}$. Entretanto, um dos critérios para incluir a revista no Current Contents é a internacionalidade ${ }^{20}$. Portanto há pressão por internacionalidade, tanto por parte de fontes indexadoras ${ }^{20}$, e de agências de pesquisas de países que não são angloparlantes ${ }^{19,51}$. Portanto é compreensível que grande número de publicações médicas nacionais e internacionais tenha optado pela publicação em inglês.

Oidioma também se relaciona ao fator de impactoe influi na dinâmica da publicação. Revistas que publicam em diferentes línguas tem perda de poder de concentração de citações, e do fator de impacto. Além disso, acrescenta mais etapas na editoração, e equivale do ponto de vista de horas de trabalho da equipe editorial e de custos, à publicação de duas revistas. Desnecessário dizer que o tempo de preparo de cada fascículo também se estende, e não seriam inesperadas repercussões negativas na qualidade editorial, pois traduçãoé uma atividade complexa. As revistas russas e japonesas que publicam simultaneamente em diferentes línguas diminuem ofator de impacto mesmo que tenham publicações relevantes ${ }^{9}$. Esseé o modelo que foi escolhido atualmente pelos Arquivos Brasileiros de Cardiologia, reiterado recentemente pela diretoria da Sociedade Brasileira de Cardiologia ${ }^{52}$.

Entre as referências dos artigos dos Arquivos predominam obviamente as referências internacionais. Cabe entretanto a indagação sobre eventual subvalorização dos dados nacionais. Embora a razão atual referências internacionais/nacionais seja inferior a 8,6 do período $1968-1977^{\text {I }}$, ela permanece elevada: as diferenças entre as médias foi crescente nos períodos trimestral $(13,3)$, bimestral $(13,7)$ e mensal (17,5) (tab. VI). A pouca representatividade dos Arquivos Brasileiros de Cardiologia como fonte de referência já foi salientada ${ }^{1}$, e demanda conscientização adicional. Muito recentemente, uma carta elegante publicada no Journal of the American College of Cardiology ${ }^{53}$ resgatou dados de uma publicação dos Arquivos Brasileiros de Cardiologia em 1951. Outra consequiência prática é que os Arquivos ficam com um baixoíndice de impacto; uma das explicações de funcionários graduados de fontes indexadoras internacionais é que a primeira razão para tanto é que os autores brasileiros não se esmeram em citar publicações nacionais [Cardoso SC, comunicação pessoal.]

Concluindo, as tendências verificadas com base nos dados que analisamos, demonstram a existência de hiatos que representam oportunidades de crescimento cultural para os Arquivos Brasileiros de Cardiologia, tão necessário para atender outros pré-requisitos internacionais de editoração e publicação de revistas médicas, melhorar a sua reputação científica, e ser instrumento do progresso da cultura médica cardiológica brasileira.

\section{Agradecimentos}

À Sra. Glória Cardoso, assessora executiva dos Arquivos Brasileiros de Cardiologia, pelos dados sobre a dinâmica das publicações, e à sra. Sueli Campos Cardoso, do Serviço de Biblioteca e Documentação da Faculdade de Medicina da USP, pelas consultas sobre referências e fontes de indexação.

\section{Referências}

1. Amorim DS. Considerações sobre as publicações de Arquivos Brasileiros de Cardiologia no período de 1968-1977. Arq Bras Cardiol 1980; 34: 1-7.

2. Barbosa MM, Paola AAV. Perfil acadêmico do cardiologista da Sociedade Brasileira de Cardiologia: um estudo de 1750 cardiologistas. Arq Bras Cardiol 1999; 73(supl IV): 54

3. McDermott MM, Lefevre F, Feinglass J, et al. Changes in study design, gender issues, and other characteristics of clinical research published in three major medical journals from 1971 to 1991. J Gen Intern Med 1995; 10: 13-8.

4. Foratini OP. A tríade da publicação científica. Rev Saúde Pública 1996; 30: 3-12.

5. Smith R. Does the world need the BMJ? Br Med J 1997; 314: 1-2.

6. Smith R. What is publication? A continuum. Br Med J 1999; 318: 142.

7. Van Rooyen S, Godlee F, Evans S, BlackN, Smith R. Effect of open peer review on quality of reviews and on reviewers' recommendations: a randomised trial. $\mathrm{Br}$ Med J 1999; 318: 23-7.

8. Winker MA, Fontanarosa PB. Letters: a forum for scientific discourse. JAMA 1999; 281: 1543 .

9. Linardi PM, Coelho PMZ, Costa HMA. The "impact factor" as a criterion for the quality of scientific production is a relative, not absolute, measure. Braz J Med Biol Res 1996; 29: 555-61.

10. Anônimo. Editorial. Arq Bras Cardiol 1948; 1: página não numerada

11. Horton R. A physician's life. Br Med J 1996; $347: 6$.

12. Vandenbroucke JP. Medical journals and the shaping of medical knowledge. Lancet 1998; 352: 2001-6.

13. Goldbeck-Wood S. Evidence on peer review - scientific quality control or smokescreen? Br Med J 1999; 318: 44-5.

14. Gorbach SL. Why a clinical infectious disease journal? Clin Infect Dis 2000; 30: 1-2.

15. Goldman L, Browner W. New Year's greetings from the American Journal of Medicine. Am J Med 2000; 108: 1.
16. Glass RM, Molter J, Hwang MY. Educating patients. One-year anniversary of the JAMA patient page. JAMA 1999; 281: 1544.

17. Meneghini R. Systematization of academic and scientific affiliation, or how to prevent data on your publications from being lost in the national and international data base. Braz J Med Biol Res 1995; 28: 617-9.

18. Paola AAV, Barbosa MM. Análise das variáveis acadêmicas relacionadas à maior produtividade científica do cardiologista brasileiro. Arq Bras Cardiol 1999; 73(supl IV): 54

19. Meneghini R. The key role of collaborative work in the growth of Brazilian science in the last ten years. Scientometrics 1996; 35: 367-73.

20. Garfield E. How can impact factors be improved? Br Med J 1996; 313: 411-3.

21. Opthof T. Sense and nonsense about the impact factor. Cardiovasc Res 1997; 33: 1-7.

22. Marrugat J. Encuesta de opinión de los lectores de la Revista Española de Cardiología. Rev Esp Cardiol 1997; 50: 1-4.

23. Frank E. Authors' criteria for selecting journals. JAMA 1994; 272: 163-4.

24. Lundberg GD, Paul MC, Fritz H. A comparison of the opinions of experts and readers as to what topics a general medical journal (JAMA) should address. JAMA 1998; 280: 288-90.

25. Hall R. Heart in the $21^{\text {st }}$ century. Heart 2000; $83: 1-2$.

26. Dean AG, Dean JA, Coulombier D, et al. Epi Info, Version 6: Center of Disease Control and Prevention, Atlanta, Georgia, USA, 1994.

27. Weber EJ, Callaham ML, Wears RL, Barton C, Young G. Unpublished research from a medical specialty meeting. Why investigators fail to publish. JAMA 1998; 280: 257-9.

28. Anônimo. Editorial. Arq Bras Cardiol 1956; 9: 1-2.

29. Winker MA, Fontanarosa PB. JAMA-express: rapid peer review and publication. JAM 1999; 281: 1754-5.

30. Gorodezky M. El impacto de las publicaciones medicas. Arch Inst Cardiol Mex 1989; 59: 445-7. 
31. Goldman L, Loscalzo A. Fate of cardiology research originally published in abstract form. NEngl J Med 1980; 303: 255-9.

32. Bignall J, Horton R. Learning from stories - The Lancet's case report. Lancet 1995; 346: 1246.

33. Borgstein J. The end of the clinical anecdote. Lancet 1999; 354: 2151-2.

34. Friesinger GC. Who should be an author? J Am Coll Cardiol 1986; 8: 1240-2.

35. Bhopal R, Rankin J, McColl E, et al. The vexing question of authorship: views of researchers in a british medical faculty. Br Med J 1997; 314: 1009-12.

36. Scott T. Changing authorship system may be counterproductive (letter). Br Med J 1997; 315: 744 .

37. Shapiro DW, Wenger NS, Shapiro MF. The contributions of authors to multiauthored biomedical research papers. JAMA 1994; 271: 438-42.

38. Rennie D, Flanagin A. Authorship! Authorship! Guests, ghosts, grafters, and the two-sided coin. JAMA 1994; 271: 469-71.

39. Uniform requirements for manuscripts submitted to biomedical journals. NEngl J Med 1997; 336: 309-15.

40. Smith R. Authorship is dying: long live contributorship. Br Med J 1997; 315: 696.

41. Ezsias A. Authorship is influenced by power and departmental politics. Br Med J 1997; 315: 746

42. Fetters MD, Elwyn TS. Assessment of authorship depends on culture. Br Med J 1997; 315: 747.
43. Block AJ. Authors, take heed. Chest 1997; 112: 301-2.

44. Kauffmann R, Reyes H, Goic A. Analisis editorial de los manuscritos enviados para publicação en la Revista Médica de Chile. Rev Med Chile 1991; 119: 327-33.

45. Zulueta MA, Bordons M. La producción científica española en el área cardiovascular a través del Science Citation Index (1990-1996). Rev Esp Cardiol 1999; 52: 751-64.

46. Holm G, Angelin B, Faire U, Fagrell B, Ljunghall S. Journal of Internal Medicine and the new century. J Intern Med 2000; 247: 1-2.

47. Anônimo. Editorial. Arq Bras Cardiol 1963; 16: página não numerada.

48. Colli W, Dietrich CP, Mendonça-Previato L, Meneghini R, Souza W, Timo-Iaria C. Forum on peer reviews and editors. Ciência e Cultura 1998; 50: 14-23.

49. Vandenbroucke JP. On not being born a native speaker of English. Br Med J 1989; 298: 1461-2.

50. Bracho-Riquelme RL, Pescador-Salas N, Reyes-Romero MA. Repercusión bibliométrica de adoptar el inglês como idioma único de publicación. Rev Invest Clin 1997; 49: 369-72.

51. Bakewell D. Publish in english, or perish? Nature 1992; 356: 648.

52. Ata da 195a. reunião da diretoria da Sociedade Brasileira de Cardiologia. Rio de Janeiro (1 de abril de 2000).

53. Lotufo PA. Winter and Cardiovascular mortality (letter). J Am Coll Cardiol 1999; $34: 2150$ 